\title{
Security System Aided by Voice Fingerprint
}

\author{
Gabriel Popan \\ Technical University of Cluj-Napoca \\ Cluj-Napoca, Romania \\ popangabriel26@yahoo.com
}

\author{
Lorena Muscar \\ Signal Processing Group, Basis of \\ Electronics Department \\ Technical University of Cluj-Napoca \\ Cluj-Napoca, Romania \\ Lorena.Muscar@bel.utcluj.ro
}

\author{
Lacrimioara Grama \\ Signal Processing Group, Basis of \\ Electronics Department \\ Technical University of Cluj-Napoca \\ Cluj-Napoca, Romania \\ Lacrimioara.Grama@bel.utcluj.ro
}

\begin{abstract}
The goal of this paper is to create a security system to identify a specific person who wants to access private information or enter a building using their voice. To perform this system, we identified a database containing the audio files of the users who will be able to authenticate with this system. Several steps were sequentially performed in order to extract the characteristics of the Mel Frequency Cepstral Coefficients from the audio files. Based on the k-Nearest Neighbor algorithm with an Euclidean distance and 4 neighbors, a training model was created. Through experimental results we prove in two ways, using confusion matrix and scatter plot, that the overall voice fingerprint recognition is $\mathbf{1 0 0 \%}$, for this particular configuration.
\end{abstract}

Keywords - security system, voice fingerprint, MFCC coefficients, kNN, Euclidean distance, Classification Learner, confusion matrix, scatter plot, Biometric technologies

\section{INTRODUCTION}

Technological advances are observed and felt daily by people, which is why they are eager to explore them, to try them, and finally to purchase them. Every movement we make today is related to technology, because its development does not focus only on a certain field, but it is like a wave that captures everything.

Today's modern systems can separate someone's voice from background noise, so voice identification is possible in a crowded environment. And even if the user is sick, hoarse, or extremely tired, and his voice sounds different, voice identification systems make the error-free check, based on the spectrogram stored in the database, using specific recognition software [1].

Biometric technologies exploit the physical and emotional characteristics of the human being, such as fingerprint, iris, speech, gait, in order to differentiate people from each other. All biometric parameters differ from one individual to another, with a minimal probability that they coincide with other people, so security systems that use biometric identification provide a higher level of security [2].

The way in which a biometric identification system works is according to the following principle: this it takes over with the help of sensors the elements that characterize the person in question, more precisely the fingerprint, face shape or voice, this all these parameters will be stored in a database in the form of codes [3]. A major advantage of these biometric identification systems is the speed with which the whole process takes place.
In Section II, the database used and how the features extraction and the classification phases are done, are shortly presented. The experimental results are the subject of Section III, while in Section IV the conclusions are dragged.

\section{FRAMEWORK AND THEORETICAL FUNDAMENTALS}

Through this paper we wanted to offer people the opportunity to know the benefits of a security system based on voice fingerprint. The multitude of daily activities, the agitation around us made us more mentally vulnerable, so the use of PINs or passwords could load the user's memory, and in the worst case they could be forgotten [4]. The system we have implemented offers security and speed, two attributes that we currently consider necessary for any user.

For the application in the first phase made, we tested several training and classification algorithms to find out which is the most suitable for the data they have. With the help of the MATLAB utility and the application it has in its composition, Classification Learner [5] we managed to identify the optimal classification algorithm.

\section{A. Database}

The database used through this research is a part from the audio database used in [6]. This database contains .wav audio files, which represent voice recordings of former students from Technical University of Cluj-Napoca. We considered the students in this database as the people who will be able to authenticate based on their voice fingerprint. They want to use this voice-based system in order to be recognized and allowed access. It consists of 125 audio signals, which represents 5 audio signals/student, recorded at $16 \mathrm{kHz}$. All 125 audio signals are distributed in 2 folders, Training with 4 audio signals/student, meaning a total of 100 audio signals and Testing with 1 audio signals/student, meaning a total of 25 audio signals. From Training folder, we will extract the coefficients to create a training model and an audio file for the testing process. With the help of the audio files from the Testing folder, we will also carry out the process of identifying people. It should be noted that the recordings made are not exposed to external disturbances, therefore the extraction of coefficients is done in an optimal way. There are 25 students (01 Adrea, 02 Anca, 03 AndreiT, 04 Balint, 05 BogdanS, 06 CameliaS, 07 ClaudiuC, 08 CristianaB, 09 DanutP, 10 FlorinL, 11 LucutaM, 12 MariusO, 13 MelindaK, 14 MelindaL, 15 NicoletaB, 16 Nutica, 17 Pascariu, 18 PaulF, 19 PetruP, 20 SamoilaR, 21 SandorK, 22 SimonaS, 23 SuciuA, 24 VasileZ, 25 Zoli). 


\section{B. Feature Extraction}

As features extracted for the audio signals, we have focused on Mel Frequency Cepstral Coefficients (MFCC), delta and delta-delta values. In order to complete this, we set the path to the folder where the files are located in the program and read each one at a time. In the Command Window can be seen how each record that is read individually appears.

The process of extracting the MFCC coefficients comprises several stages. After reading the audio files, each voice signal was divided into frames, using a Hanning window with a length of 1024-points, the sampling of the windows being done periodically. The next step was to evaluate the fast Fourier transform for each frame. We used a 512-point frame overlay. Extraction of MFCC coefficients involves the interpretation of the input signal, whether it consists of real or complex numbers. Here being only real numbers, it is interpreted as a signal in the time domain and must be a column vector. Matrix columns are treated as independent audio channels [7].

The sampling frequency is $16000 \mathrm{~Hz}$ and the number of MFCC coefficients extracted is 20 , which are used to compute also the delta and delta-delta values. The first MFCC coefficient is dropped and only the next 19 are retained, followed by the delta and delta-delta values, thus we have a total of 21 features for each audio signal.

\section{Classification}

To continue the process of implementing the solution, we created a training model based on the coefficients extracted in the previous stage. The first step was to read from the table both the coefficients and the class corresponding to the name of the person to whom the audio file corresponds, which we have done using the following MATLAB functions readtable [8], table2array [9] and table2cell [10]. Then we saved all the data in a .mat file. As a classification algorithm and to create the training model we used k-Nearest Neighbor $(\mathrm{kNN})$ algorithm with the function in MATLAB fitcknn [11].

Following the getting of the training model, the output variable $\mathrm{Y}$ is returned to us as ResponseName, which is a vector of characters that includes the names of the registered persons. It can also be seen that the number of neighbors with which an individual is compared is 4 , and as a metric distance we use the Euclidean distance. In order to use the $\mathrm{kNN}$ algorithm, several steps were needed that we went through, one of them was the use of the Classification Learner application from MATLAB. Thus, we imported the data from the table in this application, using 5-fold cross validation and finally we selected that the data to be trained with all the algorithms that this utility has.

For the testing phase we proceeded similarly to the training process. We extracted the specific coefficients of the records we use for testing, and then we compared them with the training model made in the previous point. 21 features are extracted for the test phase, using a Hanning window in 1024points, after which the fast Fourier transform is applied. Finally, denoted by coef MFCC fin, is a matrix in which the coefficients are found for each record we selected.

\section{EXPERIMENTAL RESULTS}

The feature extraction phase and the classification phase were performed in MATLAB.
Before choosing $\mathrm{kNN}$ as the best classification algorithm we have performed several test using 5-fold cross validation. The results obtained are illustrated in Fig. 1. Based on these results we have observed that $\mathrm{kNN}$ best suited for our application. In the only one classification algorithm for which the accuracy is $100 \%$.

The student identification process is done by making a comparison between the coefficients of the audio signal used for the test phase and the training model obtained previously. After we have the two data, using the predict [12] function in MATLAB we can identify the person to whom the voice signal corresponds.

\begin{tabular}{|c|c|}
\hline Last change: Cubic SVM & $21 / 21$ features \\
\hline $\begin{array}{l}1.11 \text { SVM } \\
\text { Last change: Fine Gaussian SVM }\end{array}$ & $\begin{array}{r}\text { Accuracy: } 10.0 \% \\
21 / 21 \text { features }\end{array}$ \\
\hline $\begin{array}{l}1.12 \approx S V M \\
\text { Last change: Medium Gaussian SVM }\end{array}$ & $\begin{array}{r}\text { Accuracy: } 99.2 \% \\
21 / 21 \text { features }\end{array}$ \\
\hline $\begin{array}{l}1.13 \text { SVM } \\
\text { Last change: Coarse Gaussian SVM }\end{array}$ & $\begin{array}{r}\text { Accuracy: } 0.0 \% \\
21 / 21 \text { features }\end{array}$ \\
\hline $\begin{array}{l}1.14 \mathrm{KNN} \\
\text { Last change: Fine KNN }\end{array}$ & $\begin{array}{r}\text { Accuracy: } 100.0 \% \\
21 / 21 \text { features }\end{array}$ \\
\hline $\begin{array}{l}1.15 \text { KNN } \\
\text { Last change: Medium KNN }\end{array}$ & $\begin{array}{r}\text { Accuracy: } 51.7 \% \\
21 / 21 \text { features }\end{array}$ \\
\hline $\begin{array}{l}1.16 \Rightarrow \mathrm{KNN} \\
\text { Last change: Coarse KNN }\end{array}$ & $\begin{array}{r}\text { Accuracy: } 0.0 \% \\
21 / 21 \text { features }\end{array}$ \\
\hline $\begin{array}{l}1.17 \quad \mathrm{KNN} \\
\text { Last change: Cosine KNN }\end{array}$ & $\begin{array}{r}\text { Accuracy: } 50.8 \% \\
21 / 21 \text { features }\end{array}$ \\
\hline $\begin{array}{l}1.18 \text { KNN } \\
\text { Last change: Cubic KNN }\end{array}$ & $\begin{array}{r}\text { Accuracy: } 52.5 \% \\
21 / 21 \text { features }\end{array}$ \\
\hline
\end{tabular}

Fig. 1. Some classification algorithms tested

To exemplify the functionality of the research we chose to create a graphical interface using the GUIs (Graphical user interfaces) [13] application of the MATLAB utility.

Fig. 2 [14] shows the components with which the user manages to perform several operations, but also to be aware of how the application works. It contains a welcome message, followed by some instructions that each user who uses this application must follow.

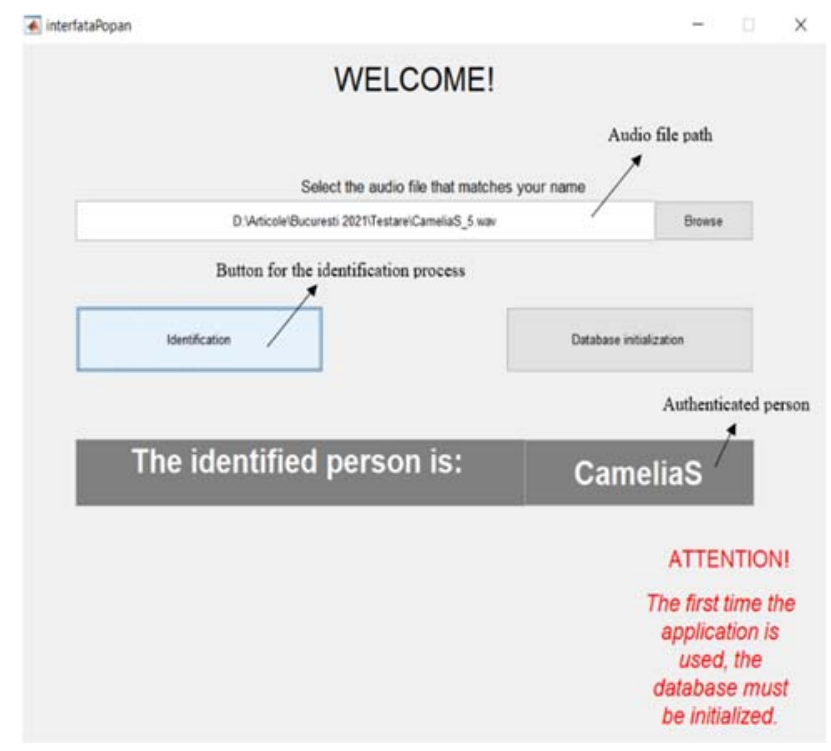

Fig. 2. Identification process [14]

The first step required for functionality is mentioned in the lower right of Fig. 2 in red, namely the initialization of the database. 


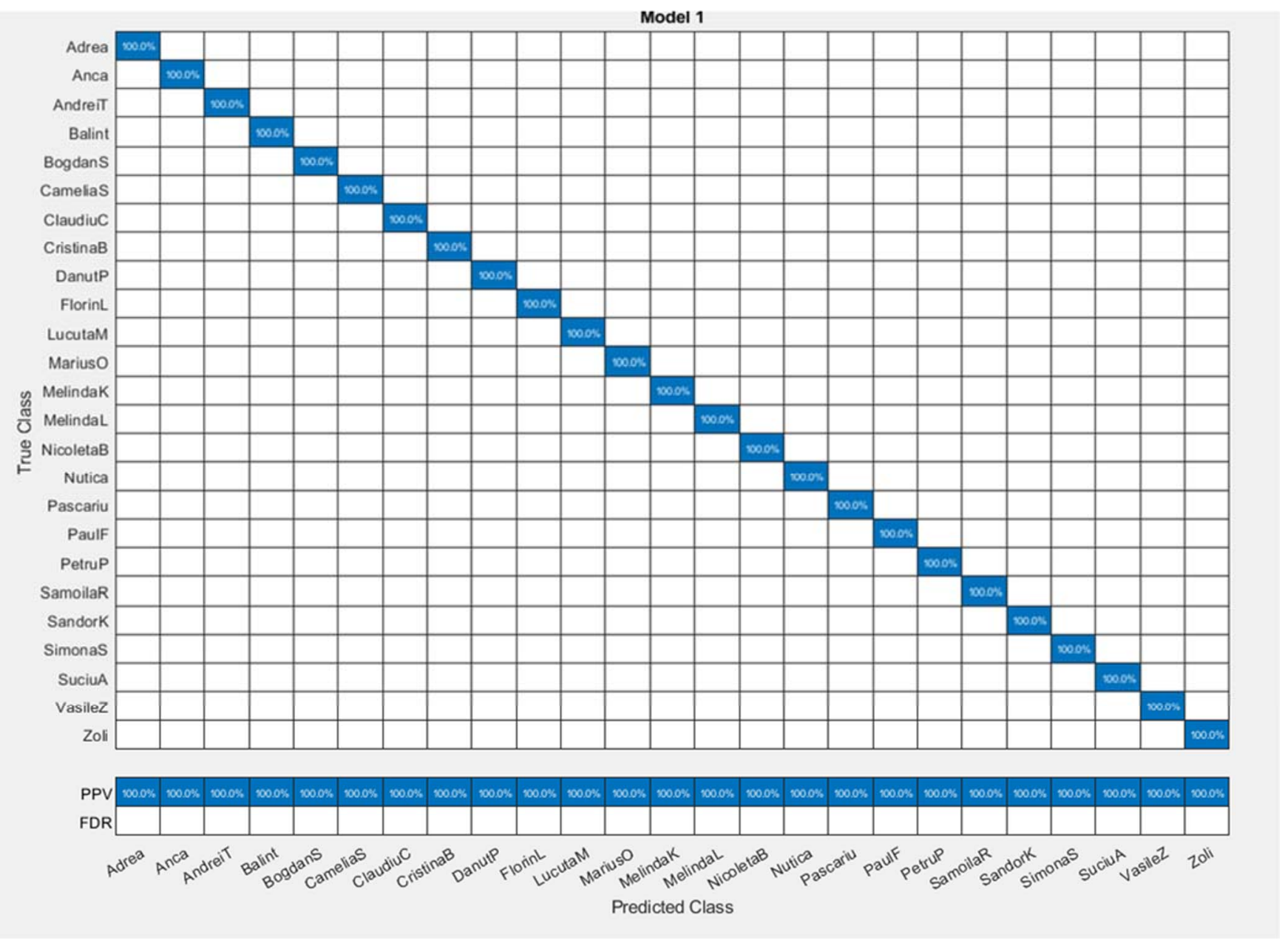

Fig. 3. Confusion matrix [14]

With the help of a push button on which is written the text "Database initialization", see Fig. 2, the initialization of the database is completed. When activated, will read the audio files from the Training folder, will extract the coefficients and create a model training that will help us on the identification part. The data we need below is assigned in two matrices, one containing the extracted coefficients and the other containing the names of the people whose audio signals are recorded.

Once the database has been initialized, the audio file corresponding to the username, one of the 25 , must be selected from the Testing folder. This is done with the help of a push button inscribed with the text "Browse". The program compares the coefficients extracted from the audio signal used for testing with the training model. After the audio file corresponding to the user's name has been selected, our path is displayed. Pressing the "Identification" button, the name of the person whose recording has been selected will appear.

Fig. 2 represents the end of the operations performed by the developed application, namely the identification of the student. We believe that this application is useful in any institution that wants a high level of security, its applicability providing confidence and stability.
With the help of audio signals, several simulations were performed in which we could observe the accuracy of the algorithm used and the degree of identification of people.

The simulations were performed using the MATLAB application, Classification Learner, where we imported the Excel document with the coefficients extracted in the training phase. Then using the kNN algorithm we trained the system and obtained the results that we will display in Fig. 3. The rows signify the True Class, and the columns show the Predicted Class. The cells on the diagonals show the percentage in which the true class and the predicted class match. If all these cells are diagonal and blue, the algorithm used for the classification process classified all the data correctly. In Fig. 3, we can also see how the classifier worked on each class, so we selected the option Positive Predictive Value (PPV) and False Discovery Rate (FDR). The first, PPV, is the probability that the subject is the tested one when restricted to the subjects who test positive, also known as precision, and FDR is the complement of PPV. The result is displayed on the bottom of the figure, here all the data are correctly classified.

The result of classifying the data with the help of the Scatter Plot is illustrated in Fig. 4. Points appear as correctly classified data, these representing the persons whose audio signals have been proposed for the training process. All the data are classified correctly, because we do not have $\mathrm{X}$ as model predictions, we obtained only points. 



Size $25 \mathrm{kB}$ Predictors: 21 Response: Var2 Response Classes: 25

Validation: 5-fold Cross-Validation

Fig. 4. Classification of data using the Scatter Plot [14]

Fig. 5 shows the accuracy of the data obtained by training the system with the $\mathrm{kNN}$ algorithm. We obtained a percentage of $100 \%$, the number of neighbors is 4 , and the distance used is the Euclidean distance. The training time is short, which gives the application speed of processing and identification.

\begin{tabular}{|c|c|c|}
\hline $1 \quad \mathrm{KNN}$ & & Accuracy: $100.0 \%$ \\
\hline Last change: Number of neigh & noors' = '4' & $21 / 21$ features \\
\hline - Current Model & & \\
\hline Model 1: Trained & & \\
\hline Results & & \\
\hline Accuracy & $100.0 \%$ & \\
\hline Total misclassification cost & 0 & \\
\hline Prediction speed & $-3400 \mathrm{obs} / \mathrm{sec}$ & \\
\hline Training time & $0.71626 \mathrm{sec}$ & \\
\hline Model Type & & \\
\hline Preset Fine KNN & & \\
\hline Number of neighbors: 4 & & \\
\hline Distance metric Euclidean & & \\
\hline Distance weight Equal & & \\
\hline Standardize data: true & & \\
\hline Optimizer Options & & \\
\hline Hyperparameter options disab & bled & \\
\hline Feature Selection & & \\
\hline All features used in the model, & 1, before PCA & \\
\hline PCA & & \\
\hline PCA disabled & & \\
\hline Misclassification Costs & & \\
\hline Cost matric default & & \\
\hline
\end{tabular}

Fig. 5. Data accuracy obtained with the kNN algorithm [14]

The ROC curve is presented in Fig. 6, showing the true positive rates and the false positive rates for the classifier that is selected in the upper right, noting that all users can be selected in turn. The red dot shows the performance of the selected classifier, in our figure it is MariusO, which has a false positive rate of 0 percent ( 0.00 displayed below the orange dot, corresponding to the $\mathrm{X}$ axis) and a true positive rate of $100 \%$ (1.00 displayed below the orange dot, corresponding $\mathrm{Y}$ axis).

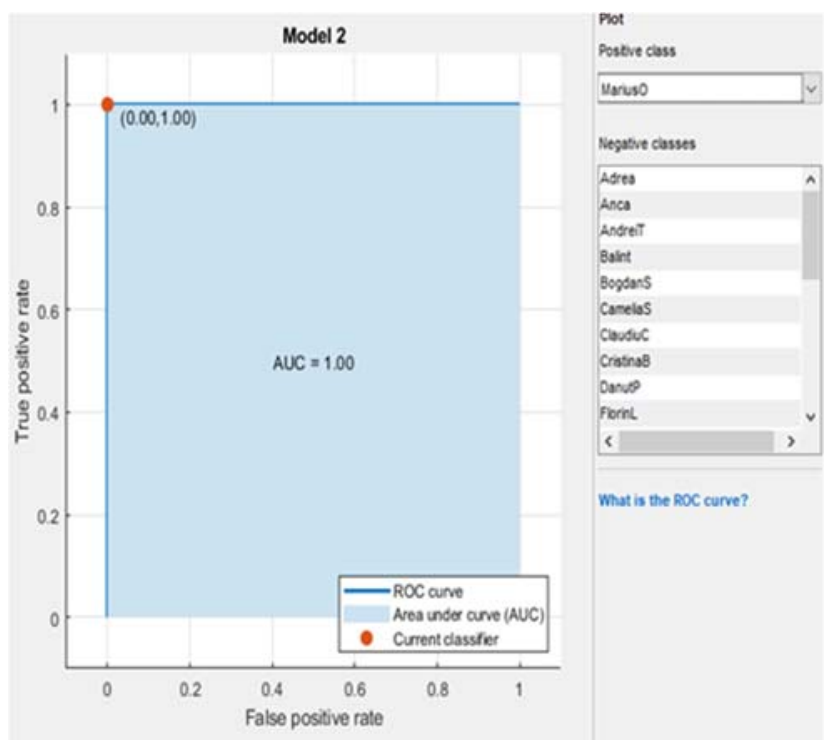

Fig. 6. ROC curve [14]

A true positive rate of 1.00 indicates that the selected classifier correctly assigns $100 \%$ of the positive class observations. A perfect result, without misclassified points, forms a right angle on the left side of the delimited square, as can be seen in Fig. 6. The area under the ROC curve, namely AUC (Area under curve) is a measure of quality of classification [15]. The larger the area is, the better the classifier performance is. 


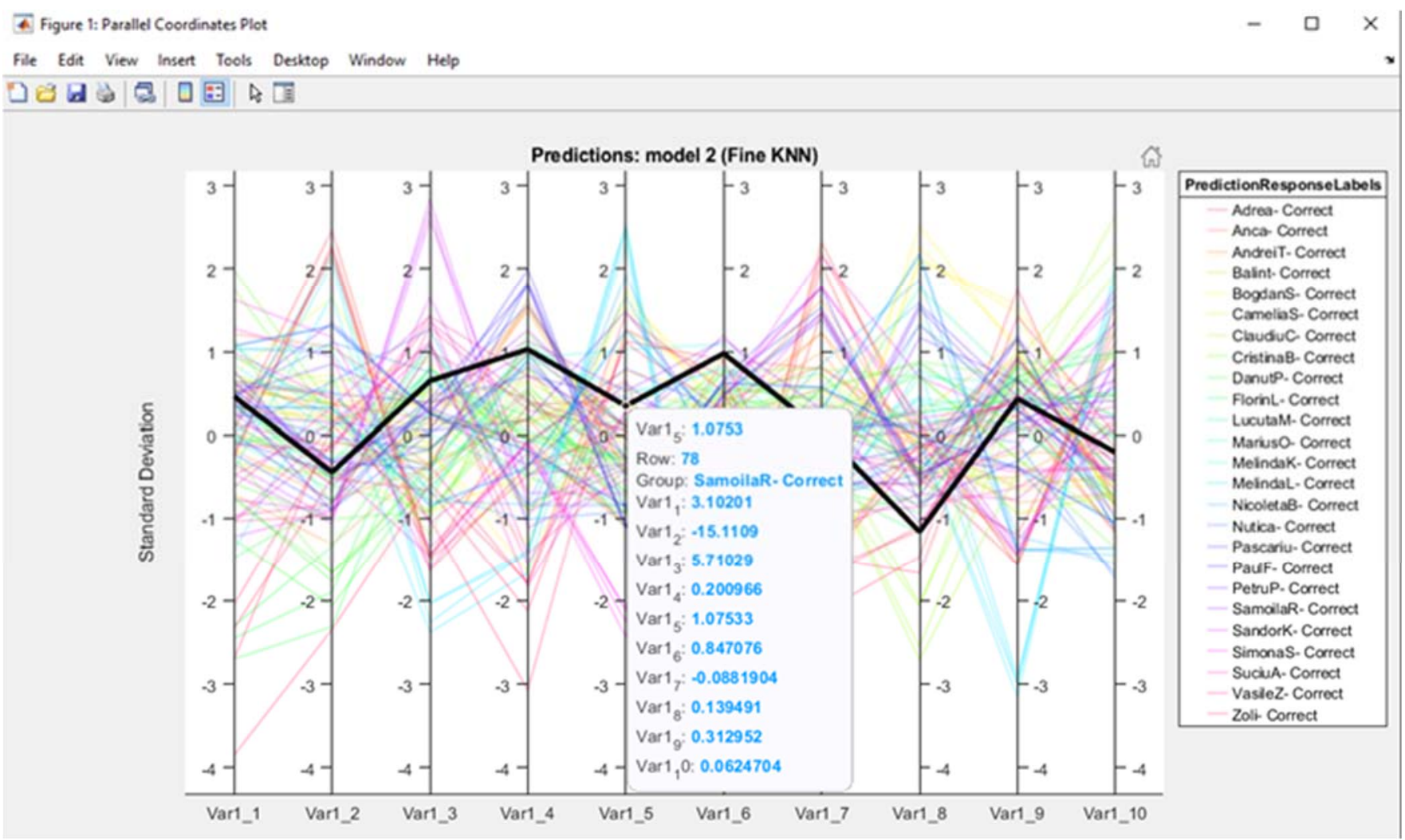

Fig. 7. Parallel coordinate diagram - Prediction Response Labels [14]

In order to be able to perform a detailed search of the users who are classified correctly, the parallel coordinate diagram is used, represented also in Fig. 7. Here the relations between the characteristics are exposed and it shows the way in which they are classified. It is observed that all the lines are continuous, which means that all the data are classified correctly, if there were broken lines, the data would not have been classified completely correctly.

\section{CONCLUSIONS}

Through this paper we have performed a security system that relies on the user's voice fingerprint. The security of personal data or the values that a person has is very important, but sometimes it can become vulnerable if we do not have a well-thought-out system at hand to protect us from possible attacks on our integrity.

The development of this system had as a starting point the identification of the database of some students, this containing their audio files. For each user, five records were taken, four of which were used for the training part, creating a training model that was compared to the fifth record, which was used for the testing part. We extracted the MFCC coefficients, the delta and the delta-delta values. We studied the results obtained from the testing process for several algorithms, these being the accuracy of the score with which the users are identified, the confusion matrix and the ROC curve. Finally, we conclude that better results are obtained for $\mathrm{kNN}$ classification algorithm.

We developed a graphical interface with which the users who use this system can interact. With its help, each person who wants to see if it is recognized by the system, will select the audio file that is named according to that person's name, and the interface will display the identified person. Finally, if the name of the audio file matches the name of the identified person, they will be allowed access to the location where this application is installed.

The applicability of security systems that are based on the voice fingerprint of users can be very wide, from access to an institution to accessing personal information. How to use it is very easy, without the need to store passwords. The way we make sounds, the shape of the mouth and the movements they make while we speak make each person's voice unique, so it is quite difficult to imitate it.

To conclude, the development of technology has also determined the need to create a more complex security system that users can use and trust.

\section{ACKNOWLEDGMENT}

This work was supported by a grant of the Romanian Ministry of Research and Innovation, CCCDI - UEFISCDI, project number 52/2020, PN-III-P2-2.1-PTE-2019-0867, within PNCDI III.

\section{REFERENCES}

[1] Dan Caragea's Blog - Biometria Vocii, 2014, https://dancarageact62.wordpress.com/2014/10/15/biometriavocii/.

[2] N. Singh, R. Khan, and R. Shree, "Applications of Speaker Recognition," in Procedia Engineering. ICMOC, 2012, pp. 22-26.

[3] D.M. Graeme, "Speaker Identification - Prototype Development and Performance," M. Eng. project, Faculty of Engineering and Surveying, Toowoomba, Australia, Jul. 2013.

[4] P. Ghosh, R. Dutta, "A new approach towards Biometric Authentication System in Palm Vein Domain," Beyond Limits, vol. 1, pp. 9-17, Mar-Apr 2012.

[5] T. Pokorny and J. Tesarik, "Microwave Stroke Detection and Classification Using Different Methods from MATLAB's 
Classification Learner Toolbox," 2019 European Microwave Conference in Central Europe (EuMCE), 2019, pp. 500-503.

[6] C. Rusu, L. Grama, "Spectrograms, Sparsograms and Spectral Signatures for Wildlife Intruder Detection," 8th International Conference on Speech Technology and Human-Computer Dialogue (SpeD), pp. 1-4, Oct. 14-17, 2015, Bucharest, Romania.

[7] Compute Mel Frequency Cepstral Coefficients in mfcc Toolbox Help, Matlab R2020b.

[8] Khalid K. Al-jabery, T. Obafemi-Ajayi, Gayla R. Olbricht, Donald C. Wunsch II, "Data analysis and machine learning tools in MATLAB and Python", Computational Learning Approaches to Data Analytics in Biomedical Applications, Academic Press, pp. 231-290, 2020.

[9] E. Lee Nylen, P.Wallisch, "Dimensionality Reduction", chapter 8, Neural Data Science, Academic Press, pp. 223 - 248, 2017.

[10] A. Das, "Kaggle - Predict survival on the Titanic challenge in MATLAB”, Electrical and Computer Engineering, Carnegie Mellon University, Jan., 2020.

[11] A. Mikhchi, M. Honarvar, N. Emam J. Kashan, M. Aminafshar, "Assessing and comparison of different machine learning methods in parent-offspring trios for genotype imputation", Journal of Theoretical Biology, Volume 399, pp. 148-158, 2016.

[12] S. Hrehova, "Predictive Model to Evaluation Quality of the Manufacturing Process Using Matlab Tools", Procedia Engineering, Volume 149, pp. 149-154, 2016.

[13] S. He, P.Li, "A MATLAB based graphical user interface (GUI) for quickly producing widely used hydrogeochemical diagrams", Geochemistry, Volume 80, Issue 4, Supplement, 125550, 2020.

[14] G. Popan. Sistem de securitate pe baza amprentei vocale. Diploma Thesis, Technical University of Cluj Napoca, Faculty of Electronics, Telecommunication and Information Technology, July 2021.

[15] Assess Classifier Performance in Classification Learner Toolbox Help, Matlab R2020b. 